68

\title{
User requirements capture for a multimedia CSCW system
}

\author{
Janni Nielsen* Gitte Lindgaard* Lone Dirckinck-Holmfeld** \\ Morten Vendelø*, Oluf Danielsen***, Marianne Georgsen** \\ * Institute of Informatics, Copenhagen Business School \\ ** Institute of Communication, Aalborg University \\ *** Institute of Computer Science, Learning, Roskilde University \\ Denmark \\ janni.nielsen@cbs.dk
}

\begin{abstract}
The early stages of a Europen Union funded multimedia CSCW project scheduled to run for several years are described. The project, called Manicoral, concerns the definition, design, development and implementation of a CSCW as well as a study of cultural, collaborative and communications aspects of a scientific community to whom the interaction media are new. The $\mathrm{CSCW}$ system encompasses both data visualisation and communications tools. These are being developed iteratively together with user requirements which are expected to change as the project matures and the user groups become accustomed to using the tools. The Scandinavian perspective and Human Factors analyses are broughttogether in a wholistic conceptual framework in the project to cover both technical requirements and collaboration, communication and cultural issues. Methods applied so far and early results are reported.
\end{abstract}

KEY WORDS: Multimedia, CSCW system, user requirements, Scandinavian perspective, human factors

\section{INTRODUCTION}

Early investigations into user needs and cultural aspects characterising a European scientific research community, the future users of this multimedia CSCW system, are reported in this paper. The project is a longitudinal study of emerging commuinication patterns, learning, collaboration and cultural characteristics of a technologically sophisticated set of user groups. In addition, it aims to define, design, develop and implement the supporting CSCW system which has two major components: communication tools and data visualisation tools. The project partners and their roles and objectives are described first. This is followed by a discussion of two major traditions brought together to provide a wholistic account of user-related needs, changes and developments of tools and behaviour during the project. These perspectives are the Scandinavian and Human Factors. Investigative methods applied so far are discussed with a focus on field studies already conducted and video analysis of two kinds of sessions in which users are defining their initial requirements. Preliminary data are reported, and research to be undertaken in the near future is discussed. 


\section{MANICORAL}

\subsection{The project}

Manicoral (Multimedia And Network In COoperative Research And Learning) is an international, interdisciplinary, user-driven research and development project involving partners from Denmark, Great Britain, The Netherlands, Austria, Italy and Greece. Disciplines represented in the project include natural science, humanities, behavioural and social science as well as technology. Manicoral is funded by the European Union's 4th Frame Program, Telematics for Research. It commenced in January 1996 and is expected to run for four years. The objective is to define, develop, implement and evaluate a multimedia Computer Supported Co-operative Work System (CSCW) among a geographically distributed European research group. It is anticipated that use of $\mathrm{CSCW}$ tools will provode better access to scientific methods, results and raw data, and that this will enhance collaboration in the user groups. This should enable these to develop sophisticated products such as global gravity maps.

\subsection{The partners: User groups}

The user group, AFRICAR (Altimetry For Research In Climate And Resources) is a network of European geoscientists who investigate the use and exploitation of radar altimetry data. The AFRICAR groups have collaborated for many years through conventional technology and infrequent face-toface meetings. Their research seeks to utilise methods for measuring the distance from satellites to the ocean surface, or to the surface of ice caps. Change in the height of the ice caps may indicate climatic change. On the ocean, the location of currents and eddies may be observed, and changes in these contribute both to a basic understanding of climatic changes and to use in weather forecasting. The location of currents is also of importance for navigation and shipping, since time and fuel may be saved by following or avoiding currents: Observations made by groups such as AFRICAR are also used for gravitational field calculations. This is important for mapping resources, for example, oil, volcanic activity or deeper processes in the earth, such as earthquakes.

Vast quantities of raw data obtained from a number of sources are stored in large databases located in different geographical locations. The accuracy of the data varies, as does their format, scale and granularity. It is difficult and cumbersome to locate, recognise, diagnose and remove noisy or erroneous data from a database. In order to compare data from different sources, these must be calibrated. This is very difficult, even for data pertaining to a particular geographical area or a certain time frame. This is partly because no common standards are imposed on the data format, database interaction methods, search and retrieval mechanisms, or on ways to extract or mark subsets of data. In an effort to inspect data effectively and efficiently, raw data are transformed into visual maps, wave forms, line graphs, and so forth. A variety of non-standard data manipulation tools are being used in these processes, many of which users themselves have developed.

\subsection{The Human Communication, Collaboration, and Cognition group (HCCC)}

Researchers in the Danish HCCC group represent the humanisties as well as the behavioural and social sciences. Their task is to develop a conceptual and methodological framework for studying, analysing and specifying user requirements for a $\mathrm{CSCW}$ system for scientific cooperation. This framework will also be used for evaluating the constitutive influence of collaborative technologies on the AFRICAR research community.

\subsection{The technical group}

The task of the technical experts at the Rutherford Appleton Laboratories (RAL) in the UK is to provide a system for Distributed Cooperative Visualisation (DCV). The DCV system will be based on the user requirements specification generated by the HCCC group, and will also be building on existing visualisation systems already used by AFRICAR. Another European project, the Telematics MERCI project, will supply CSCW tools. These will be revised and enhanced iteratively throughout the duration of the Manicoral project to fine-tune the technology so as best to match users' emerging, and probably changing, requirements. 


\section{OBJECTIVES OF THE HCCC GROUP}

Manicoral is unique in that it commenced before the CSCW tools were introduced into the AFRICAR groups. This enables a longitudinal study of how an international research community evolves, of how technology may enable new ways of collaboration and change the roles of community members, and also to study the evolution of novel ways to generate, combine and interpret scientific data.

The HCCC objectives are to:

- develop a conceptual framework and methods for developing user-driven requirements and functional specifications

- study how communication and collaboration evolve in a distributed research community over time

- study the constitutive influence of CSCW technologies on a community

\subsection{Perspectives}

In order to satisfy the HCCC objectives, two major theoretical traditions are brought together. These virtually represent two ends of a philosophical and methodological continuum that spans investigative approaches ranging from the empirico-mathematical to the cultureunderstanding (see Mc Garland \& Polgar, 1994 for elaboration). The cultureunderstanding end is represented by what has been labeled the "Scendinavian" perspective, and the empirico-mathematical end is represented by a more traditional Human Factors perspective. Relevant characteristics of these are described below.

\subsection{A Scandinavian perspective}

In Scandinavia there is a long tradition for Participatory Design (PD), or user centred design, where Union representatives and participants were involved in the systems developmental process (Greenbaum \& Kyng, 1991; Ehn, 1988; Bødker Grønbæk, \& Kyng, 1993). However, this framework alone was insufficient to satisfy the myriad of factors to be studied in the development and implementation of a CSCW tool for a technologically sophisticated user group such as AFRICAR.

To take account of these factors, a new approach. Dialogue Design. is being developed. Dialogue Design differs from the traditional Danish version of PD in that all the participants are researchers and highly experienced in using advanced technologies. Dialogue Design draws on Dialogue Research tClausen, Lorenzen \& Rasmussen., 1992), a Danish research tradition focusing on mutual dialogues between researchers and project participants as the tool for development and assessment (Cronberg, Duelund, Jensen et al., 1991). The concept of dialogue is borrowed from Habermas' ideal of a dialogue free from power games (Habermas, 1987), a true dialogue with no hidden agendae, and where participants meet with open mind and with the understanding that the 'best arguments will win'. A reasonably open-ended dialogueoriented approach is taken to accommodate and even highlight cultural and disciplinary differences between the Manicoral partners. Thus the methods applied in investigating the relevant aspects of the research community are adaptable and applied in accordance with a collaborative understanding and mutual learning processes.

In the initial step, we have applied different methods to capture the inter-dependent user requirements and their visions. Requirements cannot be captured adequately without also capturing users' vision of their future work to be supported by a new system. Visions Capture aims to outline a possible future. The notion of a 'possible' future is stressed to denote the uncertainty associated with predicting any future. Any technological tool is implemented in an existing organisational culture, into a community where clearly defined, as well as unspoken, practices are the order of the day; it will be used by people who bring their organisational and idiosyncratic understandings to the interaction. These factors influence the development- and implementation process as well as later usage patterns. Design of tools is also design of work tasks, the implementation of which may require users to obtain new qualifications. Implementation of tools thus changes organisations as well. Because the technological systems are such forceful changeagents, user driven design requires rich visions and imagination, which are fundamental cognitive tools in the design process (Nielsen, 1993). 


\subsection{The Human Factors perspective}

From a human factors angle, it is important to derive user requirements specifications that can be readily translated into user interface design. Usually, one would perform a thorough User Needs Analysis (Lindgaard, 1994) which includes some kind of task analysis. However, there are no routine tasks in the present project. Therefore, alternative methods must be explored for capturing user requirements and writing functional specifications. From the human factors perspective, it is important to understand what users want to communicate with each other, how they want their data to look, how they want to be able to identify, mark, manipulate and transpose data, and what data views they want to show, compare, and discuss.

The CSCW tools provided are novel to AFRICAR interactions which adds further elements of uncertainty the the requirements specification process: users cannot state precisely what they want; no one can predict exactly what data manipulation tools will be able to provide, and new interaction and communication patterns are likely to emerge as users gain experience using the tools. For these reasons, requirements specifications will proceed through at least two major phases. In the first phase, the aim is to understand how users expect to interact using the future CSCW. Preliminary data from this phase are presented later. In the second phase, the specifications will be adjusted iteratively according to how users are found actually to interact via these tools, and as the tools themselves evolve. It is commonly found that users' requests, outlined before the technology is available, vary substantially from the ways their interactions evolve once technological support tools are provided (Lindgaard, 1995). The iterative, concurrent refinement of requirement specifications and tools is a novel approach to systems development.

In order to study the evolution of new communication patterns and forms of collaboration, data are currently being collected on existing communication patterns within AFRICAR to determine what is being communicated by whom, how often, using which technologies, and so forth. Sampling communication among members at regular intervals over the next four years should capture any changes to existing communication patterns.
In an effort to trace the constitutive influence of a CSCW tool in this community, different meeting types undertaken by AFRICAR without the CSCW tools are currently being analysed from video recordings, but results are not yet available. In the first instance, this analysis will highlight differences and similarities between such meeting types identified to date. As with communication patterns, regular sampling of meetings should allude to any changes in meeting culture, structures or participant roles occurring over time.

\section{METHODS}

The range of methods applied so far include field studies of daily practice in some of the local research communities as well as recordings of AFRICAR meetings and discussions. In addition, two types of workshops have been held to capture user requirements: a so-called vision workshop and a mind mapping worhshop These were videotaped, and the preliminary analysis is presented later. During the field studies, a range of methods were used for data capture, including observations, video recordings, in depths interviews, photographing, and dialogue discussions.

\subsection{Field studies}

The field study methodology was partly inspired by ethnographic traditions, partly by a critical hermeneutic framework, and partly by a learning approach building on planned dialogues. In order to understand the needs and visions of the AFRICAR groups as a coherent scientific community and to investigate the constitutive role of technology field studies aiamed to elucidate daily practice in local communities. Three of the six AFRICAR communities took part. These were selected to represent the geographical spread of the community as well as varying levels of technology usage, and also to contrast the research foci within AFRICAR. The field studies aimed to gain insight into similarities and differences between the various research communities.

An observations-and-interview guide was developed from the theoretical perspectives of learning, cognition and scientific knowledge cultures which have been combined in a preliminary conceptual framework (Danielsen, 
Nielsen \& Dirckinck-Holmfeld, 1996). This is not discussed further here.

The categories in the guide included descriptions of artefacts, photographs as a still life documentation of the research institutions, listing of the technological equipment (what, where and when it is used), and a description of the local group members (educational background, research focus, employment status). Also the scientific visualisations the groups worked with/produced, (graphs, topographical maps, computer-generated visualisations and animation) and observations of learning situations (interactions between members, seminars) were noted .

The local AFRICAR communities have different characteristics. In some communities the development of visualisations of scientific data using different software tools is an indispensable part of the geophysical research; in others such tools are rarely used. The number of researchers in a local AFRICAR group varies from two 12 . Some communities have recruited new members from their own students, others have recruited candidates from related disciplines. Each local research community is thus unique in some respect which has now been documented.

Observations and note-taking were used during all field studies. In one community in which all computers were located in a computer room with several PCs and work stations, activities and collaboration were sampled in this room at regular intervals. Another community had a very small room with a coffee machine. The adjacent hallway had become an informal, but central meeting place.

Video recordings were made of sessions in which AFRICAR researchers were demonstrating how they work. Video-taping was also used to document group-interviews.

In depth, semi-structured interviews were carried out key AFRICAR members. A number of topics had been formulated in preparation, based in the theoretical model and the categories from the field guide. Parts of the interview used a more conflicting technique, called the double perspective change (Nielsen, 1987) which encourages the interviewee to change perspective during the interview.

Still Life photographs were taken systematically throughout the institute, including hallways and offices. They capture the work environment and serve as memory aids of places, layouts, people and incidents.

Dialogue Discussions were used when one of the HCCC representative stayed with the family of the local AFRICAR manager and spent several hours travelling to and from work every day. This gave ample time for informal talks. A topic would be defined as important and would then be explored freely, but persistently during the drive. Immediately after arrival, field notes would be taken to summarise the dialogue.

\subsection{AFRICAR meetings}

Face to face meetings are an important part of the AFRICAR collaboration. As it is believed that the nature, frequency, content and duration of meetings is likely to change with the introduction of the $\mathrm{CSCW}$ tools, examples of these meetings have been recorded before these tools were available to enable comparisons later in the project. Preliminary analyses of these data suggest there are three distinct types of meetings. Characteristics of these are currently being studied from video recordings, note taking and collection of all documentation tabled. The outcome of this analysis is unavailable at the time of writing.

\subsection{Capturing user requirements}

Three techniques have been applied to capture initial user requirements: (1) a mind mapping workshop, (2) a face-to-face discussion following hands-on experience with the current $\mathrm{CSCW}$ tools, and (3) a vision workshop. The first two of these were captured on videotape and highlights of the human factors analysis of these are presented later. Outcomes of the vision workshop were too general to use as a basis for drafting user requirements specifications. They are thus not presented here, but a summary may be found in Vendelø (1996).

A mind mapping workshop conducted in two sessions on consecutive days was being held at the outset of the Manicoral project to explore the possibilities for a joint project, and to identify a possible focus for the work within and between scientific groups. Mind mapping enables one to sketch and acquire an overall view of a given problem field. Participants work in small groups. In the first of two phases, the groups are asked to draw, sketch or write key concepts as they come to mind. All inputs are equal, but rationales may be 
requested. In the second phase, participants are asked to organise and add infrastructure to the concepts noted in phase 1 , resulting in a map showing goals and relationships between the concepts discussed.

Just prior to the vision workshop, a handson session of the current CSCW tools was conducted. This was followed by a group discussion focusing on the perceived merits and shortcomings of these existing tools. Some of the AFRICAR members had prepared a number of subject-specific themes to be discussed during the session and which were typical for their scientific work. Their task was to collaborate on the themes while trying out the CSCW tools. With this experience fresh in mind, the videotaped discussion proved to be an excellent source for obtaining relevant user requirements. Selected data from the analysis are presented later.

\section{RESULTS}

\subsection{Field studies}

The analysis of field study data revealed a number of dimensions along which the three communities varied. These are outlined below:

Institution: Members of the AFRICAR groups work in traditional European universities or in government research laboratories.

Politics: Ranges from geodesy and geophysics being barely tolerated to these being high priority fields

Local meetings: Colloquia and regular seminars are held in some communities; irregular discussions only in others

Teaching obligations: Ranges from none up to presenting eight lectures per week in areas such as surveying; teaching is at undergraduate and/or graduate levels

Local communities: The size of the local groups varies from two to eight senior, and a small number of junior scientists working together. In some cases, the entire group is located on a single floor; in others they are placed in different buildings.

Academic background: Geodesy, geophysics, mathematics, physics, and engineering

History of collaboration: Ranges from 24 years to recent arrivals

Publication record: Ranges from editorial board membership and frequent international publications to none
Scientific focus: Ranges from empirical estimates of satellite positioning to purely theoretical work and applied geodesy

Organisation of technology: Computers are either in common computer rooms or in people's offices. the amount of equipment varies from a PC to large work stations, plotters and other advanced machines.

Role of visualisations: Some work requires no visualisations although they may have illustrative value, whereas visualisations are central to other kinds of work

Visions for AFRICAR: AFRICAR is seen by some as a data sharing group and by others as a forum for scientific discussion and collaboration as well as specification of joint projects.

\subsection{Human factors videotape analysis}

Three videotapes were analysed, in the first instance by transwcribing all utterances spoken and the time at which they occurred. Two of the tapes were recordings from the mind mapping workshop (November 1994), and the third recorded the post-CSCW interaction meeting (March, 1996). The duration of meetings varied with the first mind mapping workshop (MM1) taking 90 minutes, the second one (MM2) taking 110 minutes, and the CSCW discussion (CSCW) taking 60 minutes respectively. Space does not allow reporting of these results in detail. Attention will therefore be brought only to the most important highlights. First, the number of unique ideas concerning a relevant aspect of the technology, possible common AFRICAR projects, and future interactions were ascertained for each meeting. These totalled 63 at MM1, 54 at MM2, and 44 at the CSCW meeting. It is not surprising that the group runs out of new suggestions over time; on that score alone, one would expect the most recent meeting to be least productive. The data appear, superficially, to support that contention.

Yet, it is interesting to note that, despite some similarities between meetings, the focus clearly shifted from one meeting to the next. At all meetings, between seven and ten topics were discussed, and roughly a quarter of the time was devoted to discussing possible data manipulation tools for the future (MM1 $27.17 \%$, MM2 $25.76 \%$, CSCW $27.17 \%$ of the meeting time). However, at MM1, the 
first $28.24 \%$ of the time was spent just getting the discussion going and ideas emerging. People clearly felt embarrassed and awkward about using this method for generating and discussing ideas. However, once they became involved, the discussion flowed freely. At MM2, roughly one third $(30.18 \%)$ of the meeting time was devoted to discussing possible projects AFRICAR might undertake in the course of the Manicoral project. This subject had not been broached in MM1 at all. In the CSCW meeting, close to one half of the time $(44.05 \%)$ was spent talking about possible ways to conduct meetings using the tools. This compares with $5.40 \%$ and $6.49 \%$ of time devoted to that topic at MM1 and MM2 respectively. At the $\mathrm{CSCW}$ meeting, one might have expected members to concentrate on the scientific themes in the ensuing discussion. Instead, it is striking that was the human-human interaction and the barriers to same caused by the technology that occupied members the most directly following this experience rather than discussing the technological merits of the tool.

In terms of deriving actual user requirements for the CSCW tools, a high proportion of the ideas tabled at the three meetings were bearing directly on specifying what users would like to be able to do interactively. At MM1 such technological ideas comprised $55.56 \%$ of the 63 ideas; at MM2 this figure was $64.81 \%$ of the 54 ideas, and at the $\mathrm{CSCW}$, it was $65.09 \%$ of the total of 44 ideas. At the time of writing the data ae still being calibrated to determine exactly how many of these technical ideas and suggestions are unique. It is expected that there will be a certain amount of overlap between the three data sets. However, it is clear already now that, in MM1 and MM2, that is before they had been exposed to the CSCW tool, users were expressing their ideas in much broader terms (eg. "it would be nice to be able to see the relationship between different data and data formats layered in one visualisation") than was the case in the $\mathrm{CSCW}$ session ("We want two overhead projectors"). Ideas in this latter case were to a large extent constrained by the technology in its current form. Depending on the amount of overlap of ideas yet to be determined, this could support the notion that users of future technology should generate their initial ideas away from, and thus unconstrained by, the technology.
The final data analysis should shed light on this argument.

\section{CONCLUSION AND FUTURE WORK}

The preliminary data provided here show that Manicoral provides an excellent opportunity for deriving user requirements before users have been exposed to the technology, and also to develop new investigative tools to accomplish this. In addition to the usual human factors analyses designed to specify the tool per se, the project also offers an opportunity to trace ongoing, probably changing, communication and interaction patterns, learning and cooperation in an emerging scientific community. In the near future, scenarios will be drafted and explored for feasibility with the users. These scenarios will be based on users' expressed wishes taken ad verbatim from the video tapes. The scenarios will probably need to specify short-term, medium and long-term realisations which will then serve to guide the further development of the tool together with other observations. The scenarios will, in the short term, be central to a participatory design workshop to be conducted soon.

\section{REFERENCES}

Clausen, C., Lorentzen, B. \& Rasmussen, L. B (eds.) (1992). Deltagelse $i$ teknologisk udvikling, Fremad, Copenhagen.

Cronberg, T, Duelund, Jensen, \& Qvortrup, L. (eds.) (1991). Danish Experiments - Social Constructions of Technolog,. Copenhagen: New Social Science Monographs

Danielsen, O. Nielsen, J. \& DirckinckHolmfeld, L. (1996). Conceptual Framework introductory reflections upon fundamental theoretical concepts in Manicoral, Manicoral, Working Paper-Deliverable 3.1

Greenbaum, J. \& Kyng, M. (1991). Introduction: Situated Design, in J. Greenbaum and M. Kyng (eds.), Cooperative Design of Computer Systems, Lawrence Erlbaum Ass. London, pp 1-25. 
Habermas, J. (1987). The Theory of Communicative Actions, Policy Press, Cambridge, vol I \& II,.

Lindgaard, G. (1995). WOW - 568 smart features on your PABX: What reallydetermines the uptake of technology? Proceedings HFT '93 International Symposium Human Factors in Telecommunications, Darmstadt, Germany, May 11-14.

Lindgaard, G. (1994). Usability testing and system evaluation: $A$ guide for designing useful computer systems, Chapman \& Hall, London.
McGarland, M. \& Polgar, S. (1994). Paradigm collapse in psychology: The necessity for a "two methods" approach, Australian Psychologist, 29 (1), 21-28.

Nielsen, J. (1987). Datamater og erkendelsesprocesser - en teoretisk analyse $\mathbf{f}$ erkendelsesparadigmer, set $\mathrm{i}$ relation til nogle 8.klasses elevers arbejde med datamater, Danmarks Lærerhøjskole, PPP publikation nr. 42.

Vendelø, M. (1996). Work task 03.2 in MANICORAL: [ID] Index and overview of initial requirements capture, working document, Copenhagen Business School, Copenhagen. 